paediatric to adult services (Transition Partnership, 2012). Young people may lose contact with health services and their health can deteriorate (Harden, Walsh, Bandler et al., 2012).

Aims NICE Guideline 43 (National Institute for Health and Care Excellence, 2016) challenges adult services to take joint responsibility with children's services for transition. We wanted to develop local networks to encourage referral of young people with palliative care needs to an adult hospice.

Methods Our referral data 2012-15 showed one transition patient referral, with extrapolation of national figures (Fraser, Miller, Hain et al., 2012) suggesting approximately 15 people (aged 16-19) in the locality with palliative care needs. We contacted local providers of palliative care to children, including children's community nurses, children's hospice and transition manager in our clinical commissioning group. We started six-monthly multi-disciplinary team meetings to discuss, with parent/carer consent, all patients aged $14+$ known to these services who might need adult palliative care, offer joint consultations and encourage referral. We have formal terms of reference for our meetings.

Results We have had three patients referred to our service in the last year, now supported by our home care team. One attends our day unit. Advance care planning is underway with two patients. Another is waiting for a transplant. Joint home visits are planned with a further two patients. For those who do not want referral yet, we write a standard letter to inform GPs of our service and that we welcome referrals of young adults.

Conclusions With this collaboration, more young people are being referred to our service and receiving continuity of palliative care. More GPs are aware of our transition service. This intervention was straightforward to implement, has had clear benefits to a local transition service, and would be easily replicable elsewhere.

\section{P-193 WORKING TOGETHER, HOSPICE AND HOSPITAL, TO MANAGE THE ACUTELY UNWELL CHILD}

Carol Wylde, Jayne Hopewell. Brian House Children's Hospice, Blackpool, UK

\subsection{6/bmispcare-2018-hospiceabs.218}

Aim To ensure the nursing team at a children's hospice have a robust system in place to recognise and manage children who become acutely unwell whilst cared for at the hospice preventing admission to hospital.

Background Children cared for at the hospice, have complex medical conditions. They are susceptible to becoming acutely unwell. The on call rota available for medical advice within the hospice was reduced significantly so an additional source of advice was required.

Method Discussion took place between hospice and local hospital enhancing collaborative working in caring for the unwell child. The paediatrician on call rota and children's assessment unit at the hospital were made available for advice in addition to the medical team at the hospice. A policy and procedure was written for use at both locations so all were clear on roles and responsibilities. Education sessions were provided on the use of the Situation Background Assessment Recommendation (SBAR) tool to ensure a concise and familiar communication system between the two services. Paediatric Early Warning Score (PEWS) tool adapted for use within the hospice.

Findings The PEWS assisted nurses to recognise and determine the level of response to an unwell child. The SBAR tool focussed communication when seeking advice. Advice available to the nursing team has been strengthened and diversified. Enhanced communication and collaborative working between the paediatric unit and children's hospice on a daily basis, not just when a child is acutely unwell.

Conclusion The nursing team at the hospice have a comprehensive system in place enabling them:

- To quickly recognise when a child is becoming acutely unwell

- To seek advice from a robust arrangement of senior nursing or medical colleagues, 24/7, within the hospice or hospital

- Ensure a coordinated and timely response is in place to effectively manage children who become acutely unwell.

\section{P-194 DEVELOPING HOSPICE AT HOME SERVICES FOR CHILDREN AND YOUNG PEOPLE}

Kathryn Norris. Derian House Children's Hospice, Chorley, Lancashire

10.1136/bmjspcare-2018-hospiceabs.219

Background Palliative care starts at diagnosis and the focus throughout should be on quality and supporting families to have the best life experience possible (Department of Health, 2008). It is recommended that holistic assessment and thorough advanced parallel planning for each eventuality can help to give the family back some control and reduce some of the anxieties involved in end of life care (Bennett \& Ilic, 2011), however, this takes time and can be easier in familiar surroundings.

Aim To extend our current hospice at home service (which is currently focused on immediate end of life care) to offer short respite visits in the family home, earlier on in a child's condition. By visiting regularly we hope to offer ongoing support and improve outcomes for children and their families.

Methods November 2017 to January 2018: Establish criteria for admission and service delivery model. Identify families who would benefit from respite at home and that fit the criteria. January to April: Recruit staff to the team and establish paperwork to assist in the running of the service. April to June: Contact families and start to offer home visits, complete Family Agreements, Passports, risk assessments, medication charts and Allocation Tool for each family. June to September: Complete individual advance care plans in liaison with multidisciplinary team. Audit the service and gather user feedback. Anticipated results

- Increasing visits to families and positive user feedback

- Increasing referrals to in-house hospice services

- Increasing numbers of completed advance care plans

- Increasing use of the resting (cold) rooms in the hospice

- Reducing numbers of 'unexpected' deaths and thus the need for transfer to hospital or coroner's mortuary after death.

Conclusion Respite care provided by the hospice at home team would benefit the children and families in the here and now, but it could also have a huge impact on the parent, sibling and family's emotional health and well-being in the future. 